\title{
IUCrJ
}

Volume 4 (2017)

Supporting information for article:

\section{CADEE: Computer-Aided Directed Evolution of Enzymes}

Beat Anton Amrein, Fabian Steffen-Munsberg, Ireneusz Szeler, Miha Purg, Yashraj Kulkarni and Shina Caroline Lynn Kamerlin 


\section{S1. Supplementary figures}

\section{\begin{tabular}{l|l|l|l} 
Sort by $\mathrm{dG} \#$ Sort by residue index & Sort by actions \\
\hline
\end{tabular}}

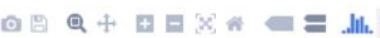

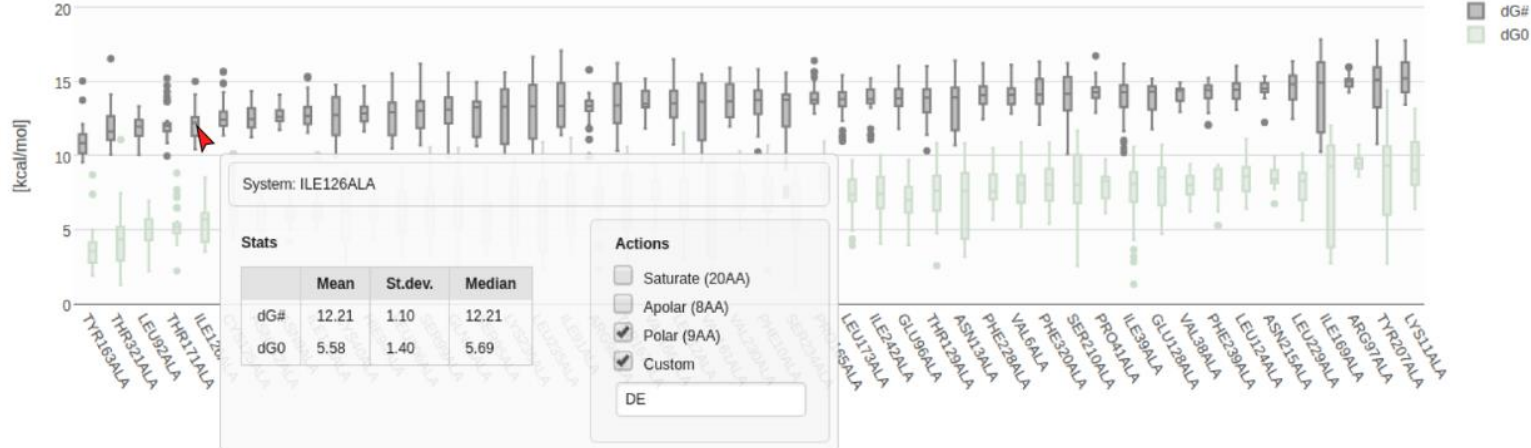

CADEE command

cadee.py --librarymut 321:SATURATE 321:POLAR 126:POLAR 126:'DE'

Figure S1 A screen shot of the CADEE graphical user interface, showing the alanine scan data presented in Fig. 8 and Table S3. The data is plotted as boxplots, with the selection tool open for illustrative purposes. The selection tool displays the option menu for the residue at position 126.
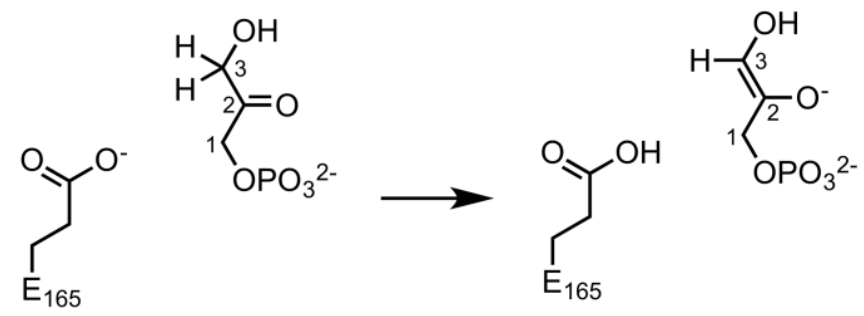

II

Figure S2 Structures of the different VB states used in this work, and the $\mathrm{C}$ atom numbering. 


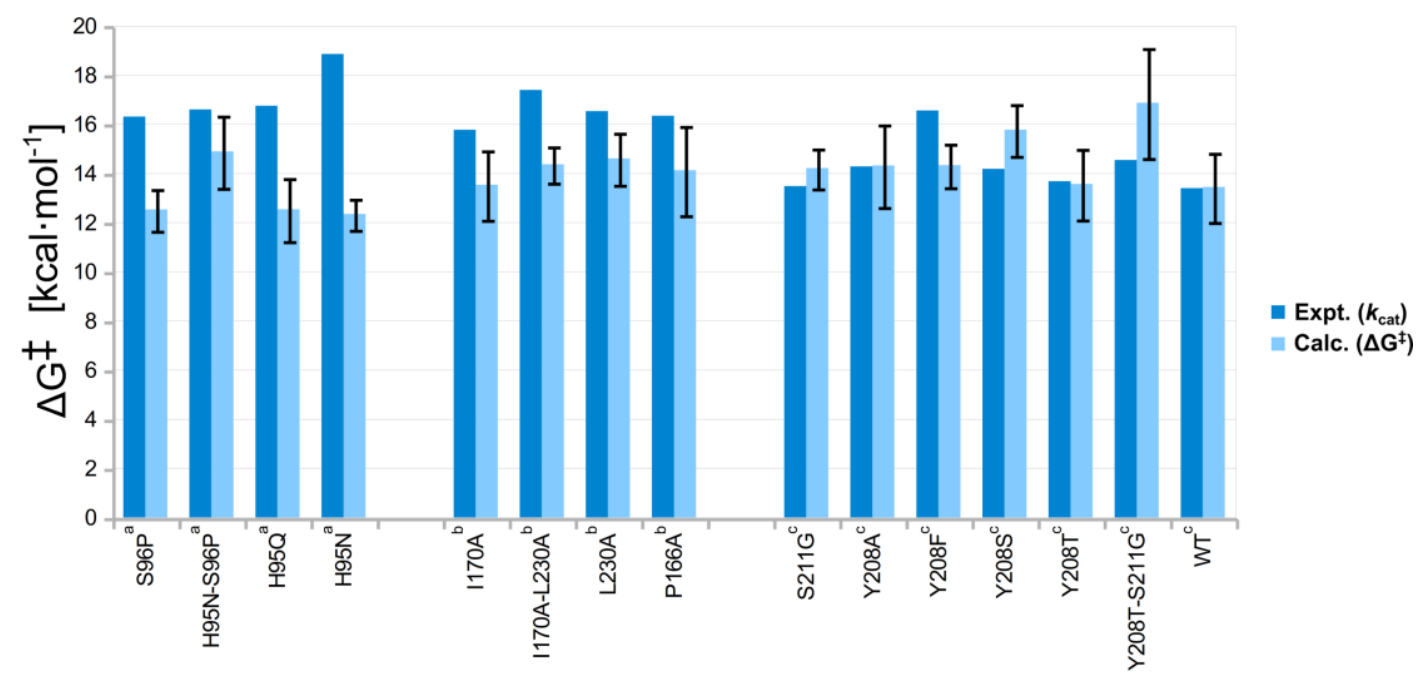

Figure S3 Experimental $\left(k_{\mathrm{cat}}\right)$ and calculated activation free energies $\left(\Delta \mathrm{G}^{\ddagger}\right)$ for the deprotonation of DHAP by diverse TIM variants (this is an extended version of Fig. 7 of the main text, now including also comparison to other organisms). The experimental data is arranged with respect to the organism and the residue numbering was adapted to the corresponding position in the Saccharomyces cerevisiae enzyme. The calculated values have all been obtained with the corresponding amino acid substitutions in the yeast enzyme, and therefore the larger discrepancy to the experimental values in these cases is therefore not surprising. It clearly illustrates that mutational effects cannot directly be transferred among enzymes from different organisms (the enzymes from chicken and Trypanosoma brucei brucei share $52 \%$ and $50 \%$ sequence identity with the yeast enzyme, respectively). Additionally, substitutions in H95, which is a catalytically critical residue in the second step (Richard, 2012), most likely lead to a drastic increase in the activation free energy in the second step as this is the step where this residue is most catalytically relevant (see mechanism in Fig. 6(b) of the main text) and thereby making it ratelimiting, which cannot be taken into account when only modelling the first step. Furthermore, the high discrepancy for the S96P mutation can be rationalized by a structural perturbation of the active site (data not shown). Note that substitutions in the chicken enzyme involve H95 which is catalytically

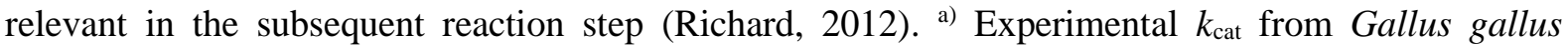
domesticus, obtained at $30^{\circ} \mathrm{C}$ (Blacklow \& Knowles, 1990). ${ }^{\text {b) }}$ Experimental $k_{\text {cat }}$ from Trypanosoma brucei brucei, obtained at $25^{\circ} \mathrm{C}$ (Malabanan et al., 2011, Zhai et al., 2013, Richard et al., 2016). c) Experimental $k_{\text {cat }}$ from Saccharomyces cerevisiae, obtained at $25{ }^{\circ} \mathrm{C}$ (Zhai et al., 2015). The corresponding data can be found in Table S2. 


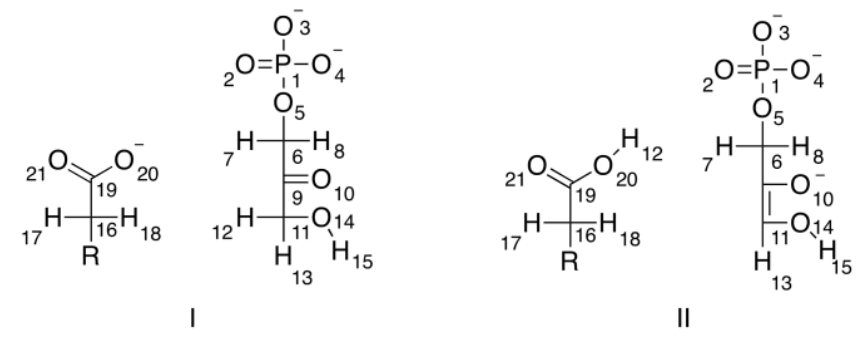

Figure S4 Atom numbering in the different VB states (corresponding to the numbering used in the EVB parameter tables provided in Section S2). 
Table S1 List of residues that were ionized in the simulations, as well as the protonation patterns of histidine residues. All other residues were outside the simulation sphere and were thus kept in their unionized forms (see also Section 2.4 of the main text).

\begin{tabular}{cc} 
Residue & Residue number \\
\hline Asp & - \\
Glu & $97,129,165,239$ \\
Lys & 12,237 \\
Arg & 98,99 \\
His- $\delta$ & $103,185,350,432$ \\
His- $\varepsilon$ & 95,342 \\
\hline
\end{tabular}


Table S2 Summary of the experimental (Expt.) and corresponding calculated (Calc.) activation free energies, $\Delta \mathrm{G}^{\ddagger}$, for various TIM variants. ${ }^{\text {a) }}$

\begin{tabular}{cccccc} 
Mutant & Expt. & Calc. & Median & $\begin{array}{c}\text { Standard } \\
\text { deviation }\end{array}$ & $\begin{array}{c}\text { Standard } \\
\text { error of } \\
\text { mean }\end{array}$ \\
\hline S96P & 16.31 & 12.53 & 12.69 & 0.85 & 0.17 \\
H95N/S96P & 16.60 & 14.89 & 14.48 & 1.46 & 0.30 \\
H95Q & 16.75 & 12.54 & 12.45 & 1.28 & 0.26 \\
H95N & 18.86 & 12.35 & 12.34 & 0.63 & 0.13 \\
& & & & & \\
I170A & 15.77 & 13.54 & 13.52 & 1.42 & 0.29 \\
I170A/L230A & 17.40 & 14.37 & 14.40 & 0.74 & 0.15 \\
L230A & 16.54 & 14.61 & 14.54 & 1.05 & 0.21 \\
P166A & 16.34 & 14.13 & 14.37 & 1.81 & 0.37 \\
& & & & & \\
S211G & 13.49 & 14.22 & 14.25 & 0.81 & 0.17 \\
Y208A & 14.29 & 14.32 & 14.15 & 1.68 & 0.34 \\
Y208F & 16.56 & 14.34 & 14.28 & 0.89 & 0.18 \\
Y208S & 14.18 & 15.78 & 15.85 & 1.05 & 0.21 \\
Y208T & 13.68 & 13.57 & 13.78 & 1.44 & 0.29 \\
Y208T/S211G & 14.55 & 16.87 & 17.54 & 2.24 & 0.46 \\
WT & 13.40 & 13.45 & 13.72 & 1.40 & 0.12
\end{tabular}

a) This data is also presented in visual form in Figs. 7 and S3. Experimental data based on (Blacklow \& Knowles, 1990, Malabanan et al., 2011, Zhai et al., 2013, Zhai et al., 2015, Richard et al., 2016). All energies are shown in $\mathrm{kcal} \cdot \mathrm{mol}^{-1}$. All calculated values presented here are averages and standard deviations / standard error of the mean over 24 individual trajectories. 
Table S3 Calculated activation free energies from the initial alanine scan. ${ }^{\text {a) }}$

\begin{tabular}{|c|c|c|c|c|c|c|c|c|c|}
\hline Mutant & Average & Median & $\begin{array}{l}\text { Standard } \\
\text { deviation }\end{array}$ & $\begin{array}{c}\text { Standard } \\
\text { error of } \\
\text { mean }\end{array}$ & Mutant & Average & Median & $\begin{array}{l}\text { Standard } \\
\text { deviation }\end{array}$ & $\begin{array}{c}\text { Standard } \\
\text { error of } \\
\text { mean }\end{array}$ \\
\hline $\mathrm{T} 322 \mathrm{~A}$ & 12.10 & 11.64 & 1.39 & 0.29 & I127A & 12.11 & 12.22 & 1.03 & 0.21 \\
\hline F321A & 14.26 & 14.10 & 1.24 & 0.25 & C126A & 12.43 & 12.12 & 1.04 & 0.21 \\
\hline $\mathrm{I} 243 \mathrm{~A}$ & 13.55 & 13.77 & 1.24 & 0.25 & L125A & 14.43 & 14.22 & 0.80 & 0.17 \\
\hline $\mathrm{F} 240 \mathrm{~A}$ & 14.11 & 14.27 & 0.87 & 0.18 & I124A & 12.84 & 12.68 & 0.81 & 0.16 \\
\hline K237A & 12.71 & 12.72 & 2.03 & 0.41 & S100A & 12.96 & 13.01 & 1.46 & 0.30 \\
\hline L236A & 13.00 & 13.34 & 1.97 & 0.40 & R99A & 13.23 & 13.37 & 1.07 & 0.22 \\
\hline $\mathrm{S} 235 \mathrm{~A}$ & 12.89 & 13.72 & 2.28 & 0.47 & R98A & 14.90 & 14.85 & 0.40 & 0.08 \\
\hline V231A & 13.90 & 13.68 & 1.26 & 0.30 & E97A & 14.00 & 13.90 & 1.01 & 0.21 \\
\hline L230A & 14.50 & 14.79 & 1.06 & 0.22 & S96A & 12.54 & 13.28 & 1.28 & 0.27 \\
\hline F229A & 14.27 & 14.21 & 0.88 & 0.18 & H95A & 12.89 & 12.81 & 0.85 & 0.18 \\
\hline $\mathrm{N} 216 \mathrm{~A}$ & 14.60 & 14.49 & 0.46 & 0.09 & L93A & 11.81 & 12.02 & 0.93 & 0.19 \\
\hline $\mathrm{N} 213 \mathrm{~A}$ & 12.62 & 12.72 & 0.76 & 0.16 & I92A & 13.57 & 13.48 & 1.63 & 0.33 \\
\hline $\mathrm{S} 211 \mathrm{~A}$ & 14.10 & 14.60 & 1.63 & 0.33 & Q64A & 12.89 & 12.83 & 1.52 & 0.31 \\
\hline Y208A & 14.79 & 15.30 & 2.30 & 0.48 & $\mathrm{P} 42 \mathrm{~A}$ & 14.42 & 14.39 & 0.81 & 0.16 \\
\hline L207A & 12.29 & 12.50 & 1.16 & 0.24 & $\mathrm{C} 41 \mathrm{~A}$ & 12.68 & 13.05 & 1.60 & 0.33 \\
\hline L174A & 13.50 & 13.84 & 1.19 & 0.24 & $\mathrm{I} 40 \mathrm{~A}$ & 13.62 & 14.11 & 1.70 & 0.35 \\
\hline $\mathrm{T} 172 \mathrm{~A}$ & 12.06 & 11.93 & 0.79 & 0.16 & V39A & 14.20 & 14.38 & 0.51 & 0.12 \\
\hline $\mathrm{I} 170 \mathrm{~A}$ & 14.43 & 15.24 & 2.28 & 0.46 & $\mathrm{~N} 14 \mathrm{~A}$ & 13.39 & 13.90 & 1.81 & 0.37 \\
\hline W168A & 13.31 & 13.48 & 1.82 & 0.37 & L13A & 13.83 & 13.77 & 1.38 & 0.28 \\
\hline V167A & 13.74 & 13.45 & 0.69 & 0.16 & $\mathrm{~K} 12 \mathrm{~A}$ & 15.24 & 15.06 & 1.17 & 0.24 \\
\hline P166A & 14.09 & 13.85 & 0.83 & 0.17 & F11A & 13.36 & 13.71 & 1.43 & 0.29 \\
\hline Y164A & 10.69 & 10.86 & 0.69 & 0.14 & $\mathrm{~N} 10 \mathrm{~A}$ & 12.63 & 12.58 & 0.56 & 0.12 \\
\hline V162A & 13.12 & 13.76 & 2.12 & 0.50 & V7A & 14.03 & 13.86 & 0.91 & 0.21 \\
\hline $\mathrm{T} 130 \mathrm{~A}$ & 13.66 & 13.93 & 1.42 & 0.29 & $\mathrm{~W}^{\prime \prime}$ & 32 & 13.72 & 4 & 0.12 \\
\hline E129A & 13.99 & 14.41 & 1.15 & 0.23 & & & $13 . / 2$ & 1.40 & 0.12 \\
\hline
\end{tabular}

a) This data is also presented in visual form in Fig. 8. All energies are shown in $\mathrm{kcal} \cdot \mathrm{mol}^{-1}$. All calculated values presented here are averages and standard deviations/standard error of the mean over 24 individual trajectories. 
Table S4 Calculated activation free energies from the separate site-saturation mutagenesis of positions 93,164 and $172 .{ }^{\text {a) }}$

\begin{tabular}{|c|c|c|c|c|c|c|c|c|c|}
\hline Mutant & Average & Median & $\begin{array}{l}\text { Standard } \\
\text { deviation }\end{array}$ & $\begin{array}{c}\text { Standard } \\
\text { error of } \\
\text { mean }\end{array}$ & Mutant & Average & Median & $\begin{array}{l}\text { Standard } \\
\text { deviation }\end{array}$ & $\begin{array}{c}\text { Standard } \\
\text { error of } \\
\text { mean }\end{array}$ \\
\hline L93A & 11.83 & 11.79 & 1.23 & 0.49 & T172N & 13.43 & 13.55 & 1.17 & 0.47 \\
\hline L93C & 13.26 & 13.00 & 0.95 & 0.38 & $\mathrm{~T} 172 \mathrm{P}$ & 14.44 & 14.20 & 0.75 & 0.30 \\
\hline L93D & 14.07 & 13.79 & 1.02 & 0.41 & T172Q & 13.50 & 13.92 & 1.90 & 0.76 \\
\hline L93E & 13.64 & 13.83 & 1.25 & 0.50 & $\mathrm{~T} 172 \mathrm{~S}$ & 14.00 & 14.61 & 1.17 & 0.47 \\
\hline L93F & 13.80 & 14.09 & 1.14 & 0.46 & $\mathrm{~T} 172 \mathrm{~V}$ & 13.80 & 14.23 & 1.20 & 0.48 \\
\hline L93G & 12.99 & 13.01 & 0.82 & 0.33 & $\mathrm{~T} 172 \mathrm{~W}$ & 13.63 & 14.04 & 1.90 & 0.76 \\
\hline L93H & 13.70 & 13.86 & 0.83 & 0.33 & $\mathrm{~T} 172 \mathrm{Y}$ & 13.43 & 13.70 & 1.39 & 0.55 \\
\hline L93I & 13.58 & 13.61 & 1.04 & 0.42 & Y164A & 11.85 & 10.89 & 2.15 & 0.86 \\
\hline L93M & 13.41 & 14.03 & 1.49 & 0.60 & $\mathrm{Y} 164 \mathrm{C}$ & 10.82 & 10.24 & 1.44 & 0.58 \\
\hline L93N & 14.64 & 14.81 & 1.40 & 0.56 & Y164D & 11.19 & 11.51 & 1.06 & 0.42 \\
\hline L93P & 12.49 & 12.43 & 0.64 & 0.25 & Y164E & 10.58 & 10.29 & 1.48 & 0.59 \\
\hline L93S & 13.30 & 13.56 & 1.32 & 0.53 & Y164F & 13.53 & 13.95 & 1.35 & 0.54 \\
\hline L93T & 12.40 & 12.35 & 1.11 & 0.44 & Y164G & 13.23 & 14.00 & 2.19 & 0.87 \\
\hline L93V & 13.20 & 13.94 & 1.85 & 0.74 & Y164H & 12.35 & 12.43 & 1.67 & 0.67 \\
\hline L93W & N.D. ${ }^{\text {b) }}$ & N.D. ${ }^{b)}$ & & & Y164I & 13.63 & 13.47 & 1.05 & 0.42 \\
\hline L93Y & 14.21 & 14.34 & 0.92 & 0.37 & Y164L & 13.21 & 14.03 & 1.94 & 0.78 \\
\hline $\mathrm{T} 172 \mathrm{~A}$ & 13.23 & 13.76 & 1.49 & 0.60 & Y164M & 13.35 & 13.93 & 1.55 & 0.62 \\
\hline $\mathrm{T} 172 \mathrm{C}$ & 14.03 & 13.96 & 0.73 & 0.29 & Y164N & 12.84 & 12.67 & 1.62 & 0.65 \\
\hline T172D & 13.04 & 13.14 & 1.95 & 0.78 & Y164P & 9.06 & 9.01 & 0.45 & 0.18 \\
\hline $\mathrm{T} 172 \mathrm{E}$ & 13.97 & 14.38 & 1.63 & 0.65 & Y164Q & 13.81 & 14.06 & 1.19 & 0.48 \\
\hline $\mathrm{T} 172 \mathrm{~F}$ & 13.95 & 14.64 & 2.20 & 0.88 & Y164S & 12.04 & 11.56 & 2.56 & 1.02 \\
\hline $\mathrm{T} 172 \mathrm{G}$ & 11.61 & 11.54 & 1.59 & 0.64 & Y164T & 14.37 & 14.35 & 0.78 & 0.31 \\
\hline $\mathrm{T} 172 \mathrm{H}$ & 13.87 & 14.30 & 1.04 & 0.42 & Y164V & 13.58 & 14.39 & 2.11 & 0.84 \\
\hline $\mathrm{T} 172 \mathrm{I}$ & 13.68 & 13.79 & 0.87 & 0.35 & Y164W & 12.46 & 12.44 & 0.82 & 0.33 \\
\hline $\mathrm{T} 172 \mathrm{~L}$ & 13.49 & 14.39 & 1.83 & 0.73 & \multirow{2}{*}{ WT } & \multirow{2}{*}{13.45} & \multirow{2}{*}{13.72} & \multirow{2}{*}{1.40} & \multirow{2}{*}{0.12} \\
\hline $\mathrm{T} 172 \mathrm{M}$ & 13.43 & 13.13 & 1.09 & 0.44 & & & & & \\
\hline
\end{tabular}

a) This data is also presented in visual form in Fig. 9. All energies are shown in $\mathrm{kcal} \cdot \mathrm{mol}^{-1}$. All calculated values presented here are averages and standard deviations/standard error of the mean over 24 individual trajectories.

b) This reaction was calculated to have extreme endothermicity, causing the transition state to disappear from the calculated free energy profile. The free energy mapping failed for these results. 
Table S5 Calculated activation free energies from the simultaneous partial saturation mutagenesis of positions 93,164 and $172 .^{\text {a) }}$

\begin{tabular}{|c|c|c|c|c|c|}
\hline $\begin{array}{c}\text { Position } \\
164 \\
\text { replacement }\end{array}$ & $\begin{array}{l}\text { Position } \\
172 \\
\text { replacem } \\
\text { ent }\end{array}$ & Average & Median & $\begin{array}{l}\text { Standard } \\
\text { deviation }\end{array}$ & $\begin{array}{c}\text { Standard } \\
\text { error of } \\
\text { mean }\end{array}$ \\
\hline \multirow{5}{*}{ Y164A } & T172D & 11.51 & 11.35 & 1.50 & 0.31 \\
\hline & $\mathrm{T} 172 \mathrm{~L}$ & 11.70 & 11.05 & 2.28 & 0.46 \\
\hline & $\mathrm{T} 172 \mathrm{R}$ & 12.64 & 12.01 & 1.71 & 0.35 \\
\hline & $\mathrm{T} 172 \mathrm{~S}$ & 10.28 & 10.01 & 1.07 & 0.22 \\
\hline & $\mathrm{T} 172 \mathrm{~W}$ & 10.63 & 10.33 & 1.00 & 0.20 \\
\hline \multirow{5}{*}{ Y164C } & T172D & 13.36 & 14.10 & 2.30 & 0.47 \\
\hline & $\mathrm{T} 172 \mathrm{~L}$ & 13.88 & 15.00 & 2.70 & 0.55 \\
\hline & $\mathrm{T} 172 \mathrm{R}$ & 11.34 & 10.87 & 1.96 & 0.40 \\
\hline & $\mathrm{T} 172 \mathrm{~S}$ & 12.75 & 12.77 & 1.65 & 0.34 \\
\hline & $\mathrm{T} 172 \mathrm{~W}$ & 11.39 & 11.13 & 2.20 & 0.45 \\
\hline \multirow{5}{*}{ Y164E } & T172D & 10.49 & 10.44 & 1.54 & 0.31 \\
\hline & $\mathrm{T} 172 \mathrm{~L}$ & 10.48 & 10.28 & 1.61 & 0.33 \\
\hline & $\mathrm{T} 172 \mathrm{R}$ & 10.94 & 11.46 & 1.80 & 0.37 \\
\hline & $\mathrm{T} 172 \mathrm{~S}$ & 10.54 & 10.35 & 1.99 & 0.41 \\
\hline & $\mathrm{T} 172 \mathrm{~W}$ & 11.90 & 11.93 & 1.25 & 0.26 \\
\hline \multirow{5}{*}{ Y164H } & T172D & 14.67 & 14.58 & 1.55 & 0.32 \\
\hline & $\mathrm{T} 172 \mathrm{~L}$ & 13.00 & 13.44 & 1.67 & 0.34 \\
\hline & $\mathrm{T} 172 \mathrm{R}$ & 13.07 & 13.36 & 0.96 & 0.20 \\
\hline & $\mathrm{T} 172 \mathrm{~S}$ & 14.05 & 13.53 & 1.93 & 0.39 \\
\hline & $\mathrm{T} 172 \mathrm{~W}$ & 13.19 & 13.84 & 1.67 & 0.34 \\
\hline \multirow{5}{*}{ Y164P } & T172D & 11.43 & 10.92 & 2.29 & 0.47 \\
\hline & $\mathrm{T} 172 \mathrm{~L}$ & 9.75 & 9.38 & 1.06 & 0.22 \\
\hline & $\mathrm{T} 172 \mathrm{R}$ & 9.92 & 9.60 & 1.26 & 0.26 \\
\hline & $\mathrm{T} 172 \mathrm{~S}$ & 10.50 & 9.23 & 2.43 & 0.50 \\
\hline & $\mathrm{T} 172 \mathrm{~W}$ & 10.92 & 10.32 & 1.93 & 0.39 \\
\hline \multirow{5}{*}{ Y164S } & T172D & 10.99 & 10.76 & 1.42 & 0.29 \\
\hline & $\mathrm{T} 172 \mathrm{~L}$ & 12.81 & 11.89 & 2.47 & 0.50 \\
\hline & $\mathrm{T} 172 \mathrm{R}$ & 12.74 & 12.11 & 1.91 & 0.39 \\
\hline & $\mathrm{T} 172 \mathrm{~S}$ & 11.05 & 9.72 & 2.52 & 0.51 \\
\hline & $\mathrm{T} 172 \mathrm{~W}$ & 11.42 & 10.34 & 2.11 & 0.43 \\
\hline WT & -- & 13.45 & 13.72 & 1.40 & 0.12 \\
\hline
\end{tabular}

a) This data, which is split into Tables S5-S8 is visualized in Fig 10. All variants of this table show L93 at position 93 . All energies are shown in $\mathrm{kcal} \cdot \mathrm{mol}^{-1}$. All calculated values presented here are averages and standard deviations/standard error of the mean over 24 individual trajectories. 
Table S6 Calculated activation free energies from the simultaneous partial saturation mutagenesis of positions 93,164 and $172 .{ }^{\text {a) }}$

\begin{tabular}{|c|c|c|c|c|c|}
\hline $\begin{array}{l}\text { Position } 164 \\
\text { replacement }\end{array}$ & $\begin{array}{l}\text { Position } 172 \\
\text { replacement }\end{array}$ & Average & Median & $\begin{array}{l}\text { Standard } \\
\text { deviation }\end{array}$ & $\begin{array}{c}\text { Standard } \\
\text { error of } \\
\text { mean }\end{array}$ \\
\hline \multirow{5}{*}{ Y164Y } & $\mathrm{T} 172 \mathrm{D}$ & 14.16 & 14.17 & 1.33 & 0.27 \\
\hline & $\mathrm{T} 172 \mathrm{~L}$ & 12.13 & 12.27 & 1.39 & 0.28 \\
\hline & $\mathrm{T} 172 \mathrm{R}$ & 12.11 & 11.90 & 0.71 & 0.15 \\
\hline & T172S & 13.58 & 13.66 & 0.81 & 0.17 \\
\hline & $\mathrm{T} 172 \mathrm{~W}$ & 12.35 & 12.42 & 1.55 & 0.32 \\
\hline \multirow{6}{*}{ Y164A } & $\mathrm{T} 172 \mathrm{~T}$ & 12.27 & 11.71 & 2.08 & 0.43 \\
\hline & T172D & 11.04 & 10.52 & 1.65 & 0.34 \\
\hline & $\mathrm{T} 172 \mathrm{~L}$ & 11.20 & 10.80 & 1.34 & 0.27 \\
\hline & $\mathrm{T} 172 \mathrm{R}$ & 10.01 & 9.87 & 1.04 & 0.21 \\
\hline & $\mathrm{T} 172 \mathrm{~S}$ & 10.71 & 10.73 & 1.01 & 0.21 \\
\hline & $\mathrm{T} 172 \mathrm{~W}$ & 10.13 & 9.85 & 1.00 & 0.20 \\
\hline \multirow{6}{*}{ Y164C } & $\mathrm{T} 172 \mathrm{~T}$ & 9.69 & 9.63 & 0.47 & 0.10 \\
\hline & T172D & 12.79 & 13.48 & 1.89 & 0.39 \\
\hline & $\mathrm{T} 172 \mathrm{~L}$ & 10.67 & 10.66 & 0.85 & 0.17 \\
\hline & $\mathrm{T} 172 \mathrm{R}$ & 11.41 & 10.14 & 2.39 & 0.49 \\
\hline & T172S & 10.64 & 10.16 & 1.56 & 0.32 \\
\hline & $\mathrm{T} 172 \mathrm{~W}$ & 10.90 & 10.95 & 1.12 & 0.23 \\
\hline \multirow{6}{*}{ Y164E } & $\mathrm{T} 172 \mathrm{~T}$ & 10.42 & 10.51 & 1.79 & 0.36 \\
\hline & T172D & 10.42 & 10.15 & 1.42 & 0.29 \\
\hline & $\mathrm{T} 172 \mathrm{~L}$ & 10.58 & 10.49 & 1.69 & 0.34 \\
\hline & $\mathrm{T} 172 \mathrm{R}$ & 9.15 & 8.90 & 1.28 & 0.26 \\
\hline & $\mathrm{T} 172 \mathrm{~S}$ & 10.24 & 9.68 & 1.52 & 0.31 \\
\hline & $\mathrm{T} 172 \mathrm{~W}$ & 9.61 & 9.48 & 1.22 & 0.25 \\
\hline \multirow{6}{*}{ Y164H } & $\mathrm{T} 172 \mathrm{~T}$ & 12.32 & 12.08 & 1.34 & 0.27 \\
\hline & $\mathrm{T} 172 \mathrm{D}$ & 13.14 & 13.00 & 0.84 & 0.17 \\
\hline & $\mathrm{T} 172 \mathrm{~L}$ & 11.56 & 10.80 & 1.54 & 0.31 \\
\hline & $\mathrm{T} 172 \mathrm{R}$ & 12.08 & 12.02 & 0.67 & 0.14 \\
\hline & $\mathrm{T} 172 \mathrm{~S}$ & 13.17 & 13.56 & 1.26 & 0.26 \\
\hline & $\mathrm{T} 172 \mathrm{~W}$ & 13.32 & 13.35 & 1.02 & 0.21 \\
\hline \multirow{6}{*}{ Y164P } & $\mathrm{T} 172 \mathrm{~T}$ & 11.76 & 11.69 & 2.72 & 0.56 \\
\hline & $\mathrm{T} 172 \mathrm{D}$ & 11.09 & 11.13 & 1.10 & 0.22 \\
\hline & $\mathrm{T} 172 \mathrm{~L}$ & 11.12 & 10.99 & 1.48 & 0.30 \\
\hline & $\mathrm{T} 172 \mathrm{R}$ & 12.32 & 12.46 & 1.51 & 0.31 \\
\hline & $\mathrm{T} 172 \mathrm{~S}$ & 10.73 & 10.05 & 1.75 & 0.36 \\
\hline & $\mathrm{T} 172 \mathrm{~W}$ & 10.95 & 10.10 & 1.70 & 0.35 \\
\hline \multirow{6}{*}{ Y164S } & $\mathrm{T} 172 \mathrm{~T}$ & 10.77 & 10.30 & 1.41 & 0.29 \\
\hline & T172D & 12.84 & 11.43 & 2.96 & 0.60 \\
\hline & $\mathrm{T} 172 \mathrm{~L}$ & 10.54 & 10.75 & 0.90 & 0.18 \\
\hline & T172R & 9.87 & 9.65 & 1.27 & 0.26 \\
\hline & $\mathrm{T} 172 \mathrm{~S}$ & 11.63 & 10.90 & 2.03 & 0.41 \\
\hline & $\mathrm{T} 172 \mathrm{~W}$ & 11.01 & 10.97 & 1.45 & 0.30 \\
\hline
\end{tabular}

a) This data, which is split into Tables S5-S8 is visualized in Fig 10. All variants shown in this table carry the L93A amino acid substitution. All energies are shown in $\mathrm{kcal} \cdot \mathrm{mol}^{-1}$. All calculated values presented here are averages and standard deviations/standard error of the mean over 24 individual trajectories. 
Table S7 Calculated activation free energies from the simultaneous partial saturation mutagenesis of positions 93,164 and $172 .{ }^{\text {a) }}$

\begin{tabular}{|c|c|c|c|c|c|}
\hline $\begin{array}{l}\text { Position } 164 \\
\text { replacement }\end{array}$ & $\begin{array}{l}\text { Position } 172 \\
\text { replacement }\end{array}$ & Average & Median & $\begin{array}{l}\text { Standard } \\
\text { deviation }\end{array}$ & $\begin{array}{c}\text { Standard } \\
\text { error of } \\
\text { mean }\end{array}$ \\
\hline \multirow{5}{*}{ Y164Y } & T172D & 12.96 & 12.73 & 2.02 & 0.41 \\
\hline & T172L & 13.06 & 13.70 & 1.82 & 0.37 \\
\hline & $\mathrm{T} 172 \mathrm{R}$ & 13.90 & 14.17 & 1.20 & 0.25 \\
\hline & $\mathrm{T} 172 \mathrm{~S}$ & 13.79 & 13.48 & 1.30 & 0.27 \\
\hline & $\mathrm{T} 172 \mathrm{~W}$ & 13.97 & 14.37 & 1.32 & 0.27 \\
\hline \multirow{6}{*}{ Y164A } & $\mathrm{T} 172 \mathrm{~T}$ & 10.83 & 10.01 & 1.80 & 0.37 \\
\hline & $\mathrm{T} 172 \mathrm{D}$ & 10.38 & 10.22 & 1.03 & 0.21 \\
\hline & T172L & 11.93 & 10.62 & 2.64 & 0.54 \\
\hline & T172R & 12.37 & 13.01 & 2.13 & 0.43 \\
\hline & $\mathrm{T} 172 \mathrm{~S}$ & 10.93 & 9.86 & 2.24 & 0.46 \\
\hline & $\mathrm{T} 172 \mathrm{~W}$ & 9.82 & 9.85 & 0.70 & 0.14 \\
\hline \multirow{6}{*}{ Y164C } & $\mathrm{T} 172 \mathrm{~T}$ & 12.28 & 12.34 & 1.71 & 0.35 \\
\hline & $\mathrm{T} 172 \mathrm{D}$ & 13.11 & 12.95 & 2.30 & 0.47 \\
\hline & $\mathrm{T} 172 \mathrm{~L}$ & 13.39 & 14.61 & 2.43 & 0.50 \\
\hline & $\mathrm{T} 172 \mathrm{R}$ & 10.89 & 10.24 & 2.18 & 0.44 \\
\hline & $\mathrm{T} 172 \mathrm{~S}$ & 13.09 & 13.77 & 2.16 & 0.44 \\
\hline & $\mathrm{T} 172 \mathrm{~W}$ & 13.11 & 13.37 & 1.59 & 0.33 \\
\hline \multirow{6}{*}{ Y164E } & $\mathrm{T} 172 \mathrm{~T}$ & 10.91 & 10.70 & 0.81 & 0.17 \\
\hline & T172D & 11.41 & 10.56 & 2.47 & 0.50 \\
\hline & T172L & 8.57 & 7.94 & 1.43 & 0.29 \\
\hline & $\mathrm{T} 172 \mathrm{R}$ & 9.93 & 9.44 & 1.74 & 0.35 \\
\hline & T172S & 10.08 & 9.81 & 1.19 & 0.24 \\
\hline & $\mathrm{T} 172 \mathrm{~W}$ & 10.40 & 10.04 & 1.29 & 0.26 \\
\hline \multirow{6}{*}{ Y164H } & $\mathrm{T} 172 \mathrm{~T}$ & 12.54 & 12.26 & 1.23 & 0.25 \\
\hline & $\mathrm{T} 172 \mathrm{D}$ & 13.14 & 13.66 & 1.55 & 0.32 \\
\hline & T172L & 12.76 & 13.61 & 2.13 & 0.44 \\
\hline & $\mathrm{T} 172 \mathrm{R}$ & 12.31 & 12.55 & 1.47 & 0.30 \\
\hline & T172S & 13.73 & 13.19 & 2.94 & 0.60 \\
\hline & $\mathrm{T} 172 \mathrm{~W}$ & 13.90 & 13.98 & 0.57 & 0.12 \\
\hline \multirow{6}{*}{ Y164P } & $\mathrm{T} 172 \mathrm{~T}$ & 11.73 & 11.23 & 2.62 & 0.54 \\
\hline & T172D & 12.34 & 12.02 & 2.49 & 0.51 \\
\hline & $\mathrm{T} 172 \mathrm{~L}$ & 10.20 & 9.71 & 1.36 & 0.28 \\
\hline & $\mathrm{T} 172 \mathrm{R}$ & 11.34 & 10.94 & 2.04 & 0.42 \\
\hline & T172S & 10.78 & 10.94 & 0.85 & 0.17 \\
\hline & $\mathrm{T} 172 \mathrm{~W}$ & 9.85 & 9.80 & 0.89 & 0.18 \\
\hline \multirow{6}{*}{ Y164S } & T172T & 10.22 & 10.24 & 0.75 & 0.15 \\
\hline & T172D & 10.72 & 10.52 & 1.52 & 0.31 \\
\hline & T172L & 10.91 & 11.04 & 1.32 & 0.27 \\
\hline & T172R & 11.89 & 11.14 & 2.78 & 0.57 \\
\hline & $\mathrm{T} 172 \mathrm{~S}$ & 10.35 & 10.57 & 0.75 & 0.15 \\
\hline & $\mathrm{T} 172 \mathrm{~W}$ & 12.13 & 11.45 & 2.55 & 0.52 \\
\hline
\end{tabular}

a) This data, which is split into Tables S5-S8 is visualized in Fig 10. All variants of this table show G93 at position 93. All energies are shown in $\mathrm{kcal} \cdot \mathrm{mol}^{-1}$. All calculated values presented here are averages and standard deviations/standard error of the mean over 24 individual trajectories. 
Table S8 Calculated activation free energies from the simultaneous partial saturation mutagenesis of positions 93,164 and $172 .{ }^{\text {a) }}$

\begin{tabular}{|c|c|c|c|c|c|}
\hline $\begin{array}{l}\text { Position } 164 \\
\text { replacement }\end{array}$ & $\begin{array}{l}\text { Position } 172 \\
\text { replacement }\end{array}$ & Average & Median & $\begin{array}{l}\text { Standard } \\
\text { deviation }\end{array}$ & $\begin{array}{c}\text { Standard } \\
\text { error of } \\
\text { mean }\end{array}$ \\
\hline \multirow{5}{*}{ Y164Y } & T172D & 13.26 & 13.20 & 2.64 & 0.54 \\
\hline & T172L & 13.73 & 13.65 & 0.99 & 0.20 \\
\hline & T172R & 12.77 & 12.54 & 1.09 & 0.22 \\
\hline & T172S & 13.39 & 13.50 & 0.89 & 0.18 \\
\hline & $\mathrm{T} 172 \mathrm{~W}$ & 12.65 & 12.56 & 1.29 & 0.26 \\
\hline \multirow{6}{*}{ Y164A } & $\mathrm{T} 172 \mathrm{~T}$ & 12.09 & 11.39 & 2.40 & 0.49 \\
\hline & T172D & 10.37 & 10.26 & 0.58 & 0.12 \\
\hline & T172L & 12.91 & 13.61 & 1.51 & 0.31 \\
\hline & T172R & 11.70 & 10.34 & 2.57 & 0.52 \\
\hline & T172S & 12.39 & 12.28 & 2.04 & 0.42 \\
\hline & $\mathrm{T} 172 \mathrm{~W}$ & 10.07 & 9.92 & 0.64 & 0.13 \\
\hline \multirow{6}{*}{ Y164C } & $\mathrm{T} 172 \mathrm{~T}$ & 10.96 & 10.48 & 1.70 & 0.35 \\
\hline & T172D & 11.11 & 10.69 & 0.94 & 0.19 \\
\hline & $\mathrm{T} 172 \mathrm{~L}$ & 13.70 & 13.75 & 2.00 & 0.41 \\
\hline & T172R & 11.42 & 10.58 & 2.73 & 0.56 \\
\hline & $\mathrm{T} 172 \mathrm{~S}$ & 11.81 & 11.64 & 1.20 & 0.24 \\
\hline & $\mathrm{T} 172 \mathrm{~W}$ & 12.71 & 12.71 & 2.70 & 0.55 \\
\hline \multirow{6}{*}{ Y164E } & $\mathrm{T} 172 \mathrm{~T}$ & 11.50 & 10.88 & 1.40 & 0.29 \\
\hline & T172D & 9.75 & 9.97 & 0.89 & 0.18 \\
\hline & T172L & 10.29 & 10.15 & 1.22 & 0.25 \\
\hline & T172R & 9.89 & 9.99 & 0.94 & 0.19 \\
\hline & T172S & 10.02 & 10.15 & 1.79 & 0.36 \\
\hline & $\mathrm{T} 172 \mathrm{~W}$ & 9.96 & 10.10 & 1.24 & 0.25 \\
\hline \multirow{6}{*}{ Y164H } & $\mathrm{T} 172 \mathrm{~T}$ & 13.37 & 13.59 & 1.05 & 0.21 \\
\hline & T172D & 13.54 & 14.24 & 1.72 & 0.35 \\
\hline & T172L & 13.40 & 14.09 & 1.52 & 0.31 \\
\hline & T172R & 12.61 & 12.91 & 0.70 & 0.14 \\
\hline & T172S & 12.86 & 13.46 & 1.55 & 0.32 \\
\hline & $\mathrm{T} 172 \mathrm{~W}$ & 12.56 & 12.38 & 1.77 & 0.36 \\
\hline \multirow{6}{*}{ Y164P } & $\mathrm{T} 172 \mathrm{~T}$ & 9.98 & 9.45 & 1.30 & 0.27 \\
\hline & T172D & 11.05 & 10.99 & 1.46 & 0.30 \\
\hline & T172L & 13.38 & 13.37 & 1.62 & 0.33 \\
\hline & T172R & 10.31 & 9.60 & 1.75 & 0.36 \\
\hline & $\mathrm{T} 172 \mathrm{~S}$ & 11.06 & 11.22 & 0.88 & 0.18 \\
\hline & $\mathrm{T} 172 \mathrm{~W}$ & 10.57 & 9.58 & 2.08 & 0.42 \\
\hline \multirow{6}{*}{ Y164S } & $\mathrm{T} 172 \mathrm{~T}$ & 13.17 & 13.50 & 2.03 & 0.41 \\
\hline & T172D & 11.75 & 11.11 & 2.14 & 0.44 \\
\hline & T172L & 10.68 & 10.82 & 0.87 & 0.18 \\
\hline & T172R & 11.55 & 10.57 & 2.68 & 0.55 \\
\hline & $\mathrm{T} 172 \mathrm{~S}$ & 12.47 & 12.13 & 1.20 & 0.25 \\
\hline & $\mathrm{T} 172 \mathrm{~W}$ & 11.98 & 11.55 & 1.44 & 0.29 \\
\hline
\end{tabular}

a) This data, which is split into Tables S5-S8 is visualized in Fig 10. All variants of this table show H93 at position 93. All energies are shown in $\mathrm{kcal} \cdot \mathrm{mol}^{-1}$. All calculated values presented here are averages and standard deviations/standard error of the mean over 24 individual trajectories. 


\section{EVB Parameters}

Table S9 EVB off-diagonal element $\left(H_{\mathrm{ij}}\right)$ and gas phase shift $\left(\alpha_{\mathrm{i}}\right)$ parameters, obtained as described in the main text (see Section 2.1).

\begin{tabular}{ccc} 
Substrate & $\begin{array}{c}H_{\mathrm{ij}} \\
\left(\mathrm{kcal} \cdot \mathrm{mol}^{-1}\right)\end{array}$ & $\begin{array}{c}\alpha_{\mathrm{i}} \\
\left(\mathrm{kcal} \cdot \mathrm{mol}^{-1}\right)\end{array}$ \\
\hline DHAP & 60.0 & 229.0
\end{tabular}

Table S10 List of the atom types and Van der Waals parameters used for atoms constituting the reacting part of the system. For all atoms except reacting atoms, a standard 6-12 Lennard-Jones potential was used. In the case of the reacting atoms, which change bonding patterns between atoms $i$ and $j$, an alternate function of the form $V_{\text {react }}=C_{i} C_{j} \exp \left(-\alpha_{\mathrm{I}} \alpha_{j} r_{i j}\right)$ was used to prevent artificial repulsion between these atoms as bonding patterns change. $\mathrm{r}_{\mathrm{ij}}$ denotes the distance $(\AA)$ between atoms $i$ and $j$. For atom type assignment see Table S11.

\begin{tabular}{|c|c|c|c|c|c|c|c|}
\hline Type & $\begin{array}{c}\mathrm{A}_{\mathrm{i}} \\
\left(\mathrm{kcal}^{1 / 2} .\right. \\
\left.\mathrm{mol}^{1 / 2} \AA^{6}\right)\end{array}$ & $\begin{array}{c}\mathrm{B}_{\mathrm{i}} \\
\left(\mathrm{kcal}^{1 / 2 .}\right. \\
\left.\mathrm{mol}^{1 / 2} \AA^{3}\right)\end{array}$ & $\begin{array}{c}\mathrm{C}_{\mathrm{i}} \\
(\mathrm{kcal} \cdot \\
\left.\mathrm{mol}^{-1}\right)\end{array}$ & $\begin{array}{c}\alpha_{\mathrm{i}} \\
\left(\AA^{2}\right)\end{array}$ & $\begin{array}{c}\mathrm{A}_{1-4} \\
\left(\mathrm{kcal}^{1 / 2}\right. \\
\left.\mathrm{mol}^{1 / 2} \AA^{3}\right)\end{array}$ & $\begin{array}{c}\mathrm{B}_{1-4} \\
\left(\mathrm{kcal}^{1 / 2}\right. \\
\left.\mathrm{mol}^{1 / 2} \AA^{3}\right)\end{array}$ & $\begin{array}{l}\text { Mass } \\
\text { (a.u.) }\end{array}$ \\
\hline $\mathrm{C} 2$ & 1802.24 & 34.18 & 1 & 2.5 & 1274.38 & 24.17 & 12.01 \\
\hline $\mathrm{CDH}$ & 1103.59 & 24.67 & 180 & 2.6 & 780.35 & 17.44 & 12.01 \\
\hline $\mathrm{CT}$ & 944.52 & 22.03 & 91 & 2.5 & 667.88 & 15.58 & 12.01 \\
\hline HA & 69.58 & 4.91 & 1 & 2.5 & 49.20 & 3.47 & 1.01 \\
\hline $\mathrm{HC}$ & 84.57 & 5.41 & 5 & 2.5 & 59.80 & 3.83 & 1.01 \\
\hline $\mathrm{HO}$ & 0.00 & 0.00 & 5 & 2.5 & 0.00 & 0.00 & 1.01 \\
\hline $\mathrm{O}$ & 616.44 & 23.77 & 1 & 2.5 & 435.89 & 16.81 & 16.00 \\
\hline $\mathrm{O} 1$ & 616.44 & 23.77 & 1 & 2.5 & 435.89 & 16.81 & 16.00 \\
\hline $\mathrm{O} 2$ & 616.44 & 23.77 & 250 & 1.5 & 435.89 & 16.81 & 16.00 \\
\hline $\mathrm{O} 2 \mathrm{Z}$ & 873.90 & 27.96 & 1 & 2.5 & 617.94 & 19.77 & 16.00 \\
\hline ODE & 601.15 & 22.27 & 250 & 1.5 & 425.08 & 15.74 & 16.00 \\
\hline ODH & 976.93 & 31.26 & 1 & 2.5 & 690.79 & 22.10 & 16.00 \\
\hline $\mathrm{OH}$ & 760.65 & 25.05 & 1 & 2.5 & 537.86 & 17.71 & 16.00 \\
\hline OP & 445.13 & 18.25 & 1 & 2.5 & 314.76 & 12.91 & 16.00 \\
\hline $\mathrm{P}$ & 2447.79 & 46.79 & 1 & 2.5 & 1730.85 & 33.09 & 30.97 \\
\hline
\end{tabular}


Table S11 Atom types in the different VB states (as depicted in Fig. S2) of atoms constituting the reacting part of the system. See Fig. S4 for atom numbering, Table S10 for the corresponding Van der Waals parameters and Table S12 for the corresponding partial charges. For atom numbering see Fig. S4.

\begin{tabular}{ccc} 
Atom number & State I & State II \\
\hline 1 & $\mathrm{P}$ & $\mathrm{P}$ \\
2 & $\mathrm{O} 2 \mathrm{Z}$ & $\mathrm{O} 2 \mathrm{Z}$ \\
3 & $\mathrm{O} 2 \mathrm{Z}$ & $\mathrm{O} 2 \mathrm{Z}$ \\
4 & $\mathrm{O} 2 \mathrm{Z}$ & $\mathrm{O} 2 \mathrm{Z}$ \\
5 & $\mathrm{OP}$ & $\mathrm{OP}$ \\
6 & $\mathrm{CT}$ & $\mathrm{CT}$ \\
7 & $\mathrm{HC}$ & $\mathrm{HC}$ \\
8 & $\mathrm{HC}$ & $\mathrm{HC}$ \\
9 & $\mathrm{C} 2$ & $\mathrm{CDH}$ \\
10 & $\mathrm{O}$ & $\mathrm{ODH}$ \\
11 & $\mathrm{CT}$ & $\mathrm{CDH}$ \\
12 & $\mathrm{HC}$ & $\mathrm{HO}$ \\
13 & $\mathrm{HC}$ & $\mathrm{HA}$ \\
14 & $\mathrm{OH}$ & $\mathrm{OH}$ \\
15 & $\mathrm{HO}$ & $\mathrm{HO}$ \\
16 & $\mathrm{CT}$ & $\mathrm{CT}$ \\
17 & $\mathrm{HC}$ & $\mathrm{HC}$ \\
18 & $\mathrm{HC}$ & $\mathrm{HC}$ \\
19 & $\mathrm{C} 2$ & $\mathrm{C} 2$ \\
20 & $\mathrm{O} 2$ & $\mathrm{ODE}$ \\
21 & $\mathrm{O} 2$ & $\mathrm{O} 1$ \\
\hline
\end{tabular}


Table S12 Atomic partial charges in the different VB states (as depicted in Fig. S2). These were obtained according to the procedure described in Section 2.4.1 of the main text. For atom numbering see Fig. S11.

\begin{tabular}{ccc} 
Atom number & State I & State II \\
\hline 1 & 1.3336 & 1.3059 \\
2 & -0.9265 & -0.9666 \\
3 & -0.9265 & -0.9666 \\
4 & -0.9265 & -0.9666 \\
5 & -0.5731 & -0.5056 \\
6 & 0.2501 & 0.1700 \\
7 & -0.0386 & -0.0255 \\
8 & -0.0386 & -0.0255 \\
9 & 0.5084 & 0.3517 \\
10 & -0.5961 & -0.8006 \\
11 & 0.3072 & -0.4685 \\
12 & -0.0187 & 0.4500 \\
13 & -0.0187 & 0.1608 \\
14 & -0.7439 & -0.6532 \\
15 & 0.4079 & 0.3883 \\
16 & -0.2200 & -0.1200 \\
17 & 0.0600 & 0.0600 \\
18 & 0.0600 & 0.0600 \\
19 & 0.7000 & 0.5200 \\
20 & -0.8000 & -0.5300 \\
21 & -0.8000 & -0.4400 \\
& &
\end{tabular}


Table S13 Bond types and corresponding parameters for covalent bonds of the reacting part of the system. Bonds of non reacting atoms are described as harmonic potentials $\mathrm{V}_{\text {Harmonic }}=0.5 \mathrm{k}\left(\mathrm{r}_{\mathrm{ij}}-\mathrm{r}_{0}\right)^{2}$, while bonds of reacting atoms are described as Morse potentials $\mathrm{V}_{\text {Morse }}=\operatorname{De}\left\{1-\exp \left[-\alpha\left(\mathrm{r}_{\mathrm{ij}}-\mathrm{r}_{0}\right)\right]\right\}^{2}$. For bond type assignment see Table S14.

\begin{tabular}{|c|c|c|c|c|c|}
\hline Bond type & $\begin{array}{c}E_{D} \\
\left(\mathrm{kcal} \cdot \mathrm{mol}^{-1}\right)\end{array}$ & $\begin{array}{c}\alpha \\
\left(\AA^{-2}\right)\end{array}$ & $\begin{array}{l}r_{0} \\
(\AA)\end{array}$ & $\begin{array}{c}k_{b} \\
\left(\mathrm{kcal} \cdot \mathrm{mol}^{-1} . \AA^{-2}\right)\end{array}$ & $\begin{array}{l}\mathrm{b} \\
(\AA)\end{array}$ \\
\hline 0 & \multicolumn{5}{|c|}{ Not Set } \\
\hline 1 & & & & 1312 & 1.250 \\
\hline 2 & & & & 900 & 1.364 \\
\hline 3 & & & & 1140 & 1.229 \\
\hline 4 & 245.8 & 1.5 & 0.945 & & \\
\hline 5 & & & & 634 & 1.522 \\
\hline 6 & & & & 634 & 1.510 \\
\hline 7 & & & & 1140 & 1.229 \\
\hline 8 & & & & 900 & 1.370 \\
\hline 9 & & & & 1098 & 1.340 \\
\hline 10 & 85.0 & 2.0 & 1.090 & & \\
\hline 11 & & & & 680 & 1.090 \\
\hline 12 & & & & 680 & 1.080 \\
\hline 13 & & & & 640 & 1.410 \\
\hline 14 & & & & 900 & 1.370 \\
\hline 15 & & & & 1050 & 1.480 \\
\hline 16 & & & & 460 & 1.610 \\
\hline 17 & & & & 640 & 1.410 \\
\hline 18 & & & & 1106 & 0.945 \\
\hline 19 & & & & 536 & 1.529 \\
\hline
\end{tabular}


Table S14 Bond type assignment for the covalent bonds of the reacting part of the system in the different VB states (as depicted in Fig. S2). For atom numbering see Fig. S4.

\begin{tabular}{cccc}
\multicolumn{2}{c}{ Atom number } & \multicolumn{2}{c}{ Bond type } \\
$\# 1$ & $\# 2$ & State I & State II \\
\hline 1 & 2 & 15 & 15 \\
1 & 3 & 15 & 15 \\
1 & 4 & 15 & 15 \\
1 & 5 & 16 & 16 \\
5 & 6 & 17 & 17 \\
6 & 9 & 5 & 6 \\
6 & 7 & 11 & 11 \\
6 & 8 & 11 & 11 \\
9 & 10 & 7 & 8 \\
9 & 11 & 5 & 9 \\
11 & 12 & 10 & 0 \\
11 & 13 & 11 & 12 \\
11 & 14 & 13 & 14 \\
14 & 15 & 18 & 18 \\
16 & 17 & 11 & 11 \\
16 & 18 & 11 & 11 \\
16 & 19 & 5 & 5 \\
19 & 20 & 1 & 2 \\
19 & 21 & 1 & 3 \\
20 & 12 & 0 & 4 \\
$\mathrm{R}^{\mathrm{a}}$ & 16 & 19 & 19
\end{tabular}

a) $\mathrm{R}$ corresponds to $\mathrm{C}_{\beta}$ in $\mathrm{E} 165$, which has no assigned atom number as it is not part of the reacting system (the corresponding PDB atom number is used in the setup of the simulations). 
Table S15 Angle types and the corresponding parameters used for bending adjacent bonds in the reacting part of the system. The angle potential is described as $\mathrm{V}_{\text {angle }}=0.5 \Sigma k\left(\Theta-\Theta_{0}\right)^{2}$. For the assignment of angle types see Table S16.

\begin{tabular}{|c|c|c|c|c|c|}
\hline $\begin{array}{l}\text { Angle } \\
\text { type }\end{array}$ & $\begin{array}{c}k_{a} \\
\left(\mathrm{kcal} \cdot \mathrm{mol}^{-1} \cdot \mathrm{rad}^{-2}\right)\end{array}$ & $\begin{array}{l}\Theta \\
\left({ }^{\circ}\right)\end{array}$ & $\begin{array}{l}\text { Angle } \\
\text { type }\end{array}$ & $\begin{array}{c}k_{a} \\
\left(\mathrm{kcal} \cdot \mathrm{mol}^{-1} \cdot \mathrm{rad}^{-2}\right)\end{array}$ & $\begin{array}{l}\Theta \\
\left(^{\circ}\right)\end{array}$ \\
\hline 0 & \multicolumn{2}{|c|}{ Not Set } & 13 & 100.0 & 109.50 \\
\hline 1 & 140.0 & 117.00 & 14 & 140.0 & 123.00 \\
\hline 2 & 160.0 & 120.40 & 15 & 140.0 & 123.00 \\
\hline 3 & 70.0 & 113.00 & 16 & 110.0 & 108.50 \\
\hline 4 & 100.0 & 109.50 & 17 & 70.0 & 113.00 \\
\hline 5 & 140.0 & 120.00 & 18 & 66.0 & 107.80 \\
\hline 6 & 160.0 & 120.40 & 19 & 70.0 & 109.50 \\
\hline 7 & 140.0 & 123.00 & 20 & 70.0 & 114.50 \\
\hline 8 & 140.0 & 116.00 & 21 & 200.0 & 108.23 \\
\hline 9 & 140.0 & 124.00 & 22 & 200.0 & 120.50 \\
\hline 10 & 70.0 & 109.50 & 23 & 70.0 & 109.50 \\
\hline 11 & 70.0 & 109.50 & 24 & 75.0 & 110.70 \\
\hline 12 & 70.0 & 120.00 & 25 & 126.0 & 111.10 \\
\hline
\end{tabular}


Table S16 Angle type assignment in the different VB states (as depicted in Fig. S2). For atom numbering see Fig. S4.

\begin{tabular}{|c|c|c|c|c|}
\hline \multicolumn{3}{|c|}{ Atom number } & \multicolumn{2}{|c|}{ Angle type } \\
\hline$\# 1$ & $\# 2$ & $\# 3$ & State I & State II \\
\hline 1 & 5 & 6 & 22 & 22 \\
\hline 5 & 6 & 9 & 4 & 5 \\
\hline 5 & 1 & 2 & 21 & 21 \\
\hline 5 & 1 & 3 & 21 & 21 \\
\hline 5 & 1 & 4 & 21 & 21 \\
\hline 5 & 6 & 7 & 23 & 23 \\
\hline 5 & 6 & 8 & 23 & 23 \\
\hline 6 & 9 & 10 & 6 & 7 \\
\hline 6 & 9 & 11 & 8 & 9 \\
\hline 7 & 6 & 9 & 10 & 11 \\
\hline 8 & 6 & 9 & 10 & 11 \\
\hline 9 & 11 & 12 & 10 & 0 \\
\hline 9 & 11 & 13 & 10 & 12 \\
\hline 9 & 11 & 14 & 13 & 14 \\
\hline 10 & 9 & 11 & 6 & 15 \\
\hline 11 & 14 & 15 & 16 & 17 \\
\hline 12 & 11 & 13 & 18 & 0 \\
\hline 12 & 11 & 14 & 19 & 0 \\
\hline 13 & 11 & 14 & 19 & 20 \\
\hline 16 & 19 & 21 & 1 & 2 \\
\hline 17 & 16 & 19 & 10 & 10 \\
\hline 18 & 16 & 19 & 10 & 10 \\
\hline 19 & 20 & 12 & 0 & 3 \\
\hline $\mathrm{R}^{\mathrm{a})}$ & 16 & 17 & 24 & 24 \\
\hline $\mathrm{R}^{\text {a) }}$ & 16 & 18 & 24 & 24 \\
\hline $\mathrm{R}^{\text {a) }}$ & 16 & 19 & 25 & 25 \\
\hline
\end{tabular}

a) $\mathrm{R}$ corresponds to $\mathrm{C}_{\beta}$ in $\mathrm{E} 165$, which has no assigned atom number as it is not part of the reacting system (the corresponding PDB atom number is used in the setup of the simulations). 
Table S17 Torsion types and corresponding parameters used in the reacting part of the system. The torsion angle potential is described as $\mathrm{V}_{\text {torsion }}=\mathrm{V}_{1}(1+\cos (\mathrm{n} \varphi-\delta))+\mathrm{V}_{2}(1+\cos 2(\mathrm{n} \varphi-\delta))+\mathrm{V}_{3}(1+\cos 3(\mathrm{n} \varphi-\delta))$, here $n$ is the periodicity (number of maxima per turn) and $\delta$ is the phase shift. For torsion type assignment see Table S18.

\begin{tabular}{cccccccc} 
Torsion type & $\mathrm{V}_{1}$ & $\mathrm{~V}_{2}$ & $\mathrm{~V}_{3}$ & Torsion type & $\mathrm{V}_{1}$ & $\mathrm{~V}_{2}$ & $\mathrm{~V}_{3}$ \\
& 0.5. barrier height $\left(\mathrm{kcal} \cdot \mathrm{mol}^{-1}\right)$ & & 0.5 .barrier height $\left(\mathrm{kcal} \cdot \mathrm{mol}^{-1}\right)$ \\
\hline 0 & & Not Set & & 14 & 0.0000 & 7.0000 & 0.0000 \\
1 & 0.0000 & 0.2730 & 0.0000 & 15 & 0.0000 & 0.0000 & 0.0000 \\
2 & 0.0000 & 0.5830 & 0.0000 & 16 & 0.0000 & 0.0000 & 0.0000 \\
3 & 1.5000 & 2.4500 & 0.0000 & 17 & 0.0000 & 0.0000 & -0.1860 \\
4 & 0.0000 & 0.0000 & 0.0000 & 18 & -0.1780 & -0.0870 & 0.2460 \\
5 & 0.0000 & 0.0000 & 0.0000 & 19 & 0.7500 & 0.0000 & 0.0000 \\
6 & 0.0000 & 2.4500 & 0.0000 & 20 & 0.0000 & 7.0000 & 0.0000 \\
7 & -0.2750 & 0.0000 & 0.0000 & 21 & 2.1590 & 0.0000 & 0.0000 \\
8 & 2.1595 & 0.0000 & 0.0000 & 22 & 0.0000 & 7.0000 & 0.0000 \\
9 & 0.8550 & -0.2500 & 0.3310 & 23 & 0.0000 & 0.0000 & 0.2250 \\
10 & 0.2500 & 0.0000 & 0.0000 & 24 & 0.0000 & 2.7500 & 0.0000 \\
11 & 0.0000 & 0.0000 & 0.1370 & 25 & 0.0000 & 0.0000 & 0.2810 \\
12 & 0.0000 & 7.0000 & 0.0000 & 26 & 0.0000 & 0.0000 & 0.1790 \\
13 & 0.8550 & -0.2500 & 0.3310 & 27 & 0.0000 & 1.4950 & 0.0000 \\
\hline
\end{tabular}


Table S18 Torsion type assignment in the different VB states (as depicted in Fig. S2). For atom numbering see Fig. S4.

\begin{tabular}{|c|c|c|c|c|c|}
\hline \multicolumn{4}{|c|}{ Atom number } & \multicolumn{2}{|c|}{ Torsion type } \\
\hline$\# 1$ & $\# 2$ & $\# 3$ & $\# 4$ & State I & State II \\
\hline 1 & 5 & 6 & 7 & 26 & 26 \\
\hline 1 & 5 & 6 & 8 & 26 & 26 \\
\hline 1 & 5 & 6 & 9 & 27 & 27 \\
\hline 2 & 1 & 5 & 6 & 25 & 25 \\
\hline 3 & 1 & 5 & 6 & 25 & 25 \\
\hline 4 & 1 & 5 & 6 & 25 & 25 \\
\hline 5 & 6 & 9 & 10 & 7 & 8 \\
\hline 5 & 6 & 9 & 11 & 9 & 10 \\
\hline 6 & 9 & 11 & 12 & 11 & 0 \\
\hline 6 & 9 & 11 & 13 & 11 & 12 \\
\hline 6 & 9 & 11 & 14 & 13 & 14 \\
\hline 7 & 6 & 9 & 10 & 15 & 16 \\
\hline 7 & 6 & 9 & 11 & 11 & 17 \\
\hline 8 & 6 & 9 & 10 & 15 & 16 \\
\hline 8 & 6 & 9 & 11 & 11 & 17 \\
\hline 9 & 11 & 14 & 15 & 18 & 19 \\
\hline 10 & 9 & 11 & 12 & 15 & 0 \\
\hline 10 & 9 & 11 & 13 & 15 & 20 \\
\hline 10 & 9 & 11 & 14 & 21 & 22 \\
\hline 12 & 11 & 14 & 15 & 23 & 0 \\
\hline 13 & 11 & 14 & 15 & 23 & 24 \\
\hline 16 & 19 & 20 & 12 & 0 & 3 \\
\hline 17 & 16 & 19 & 21 & 4 & 5 \\
\hline 18 & 16 & 19 & 21 & 4 & 5 \\
\hline 21 & 19 & 20 & 12 & 0 & 6 \\
\hline $\mathrm{R}^{\text {a) }}$ & 16 & 19 & 20 & 1 & 2 \\
\hline $\mathrm{R}^{\mathrm{a})}$ & 16 & 19 & 21 & 1 & 1 \\
\hline
\end{tabular}

a) $R$ corresponds to $C_{\beta}$ in E165, which has no assigned atom number as it is not part of the reacting system (the corresponding PDB atom number is used in the setup of the simulations). 
Table S19 Improper torsion types and corresponding parameters. The improper torsion potential is described as $\mathrm{V}_{\text {improper }}=k\left(\tau-\tau_{0}\right)^{2}$, where $k_{i}$ is the force constant and $\tau$ is the equilibrium angle (in degrees). For torsion type assignment see Table S20.

\begin{tabular}{ccc} 
Improper type & $\begin{array}{c}k_{a} \\
\left(\mathrm{kcal} \cdot \mathrm{mol}^{-1} \mathrm{rad}^{-2}\right)\end{array}$ & $\begin{array}{c}\tau_{0} \\
\left({ }^{\circ}\right)\end{array}$ \\
\hline 0 & 15.0 & Not set \\
1 & 10.5 & 180 \\
2 & 15.0 & 180 \\
3 & \multicolumn{2}{c}{180} \\
\hline
\end{tabular}

Table S20 Improper torsion type assignment in the different VB states (as depicted in Fig. S2). For atom numbering see Fig. S4.

\begin{tabular}{cccccc} 
& \multicolumn{2}{c}{ Atom number } & \multicolumn{3}{c}{ Improper type } \\
$\# 1$ & $\# 2$ & $\# 3$ & $\# 4$ & State I & State II \\
\hline 9 & 11 & 13 & 14 & 0 & 1 \\
10 & 9 & 6 & 11 & 2 & 3 \\
16 & 19 & 20 & 21 & 2 & 2 \\
\hline
\end{tabular}




\section{References}

Blacklow, S. C. \& Knowles, J. R. (1990) Biochemistry 29, 4099-4108.

Malabanan, M. M., Amyes, T. L. \& Richard, J. P. (2011) J. Am. Chem. Soc. 133, 16428-16431.

Richard, J. P. (2012). Biochemistry 51, 2652-2661.

Richard, J. P., Amyes, T. L., Malabanan, M. M., Zhai, X., Kim, K. J., Reinhardt, C. J., Wierenga, R. K., Drake, E. J. \& Gulick, A. M. (2016) Biochemistry 55, 3036-3047.

Zhai, X., Amyes, T. L. \& Richard, J. P. (2015) J. Am. Chem. Soc. 137, 15185-15197.

Zhai, X., Amyes, T. L., Wierenga, R. K., Loria, J. P. \& Richard, J. P. (2013) Biochemistry 52, 59285940. 